\title{
Migrantes provenientes de Venezuela en el Área Metropolitana de Bucaramanga 2018 - 2021
}

\section{Venezuelan migrants in Bucaramanga Metropolitan Area}

\author{
Maria Eugenia Bonilla Ovallos (iD \\ Universidad Autónoma de Bucaramanga, Colombia \\ mbonilla566@unab.edu.co
}

\section{Maria Lucía Rivero Arenas (iD}

Universidad Autónoma de Bucaramanga, Colombia

mrivero674@unab.edu.co

Nadia Pérez Guevara (iD

Universidad Autónoma de Bucaramanga, Colombia

nperez491@unab.edu.co

\author{
Cómo citar este artículo: \\ Bonilla, M., Rivero, M. \& Pérez Guevara, N. (2021). Migrantes provenientes de Venezuela en el Área Metropolitana de \\ Bucaramanga 2018 - 2021. Reflexión Política 23(48), pp. 6-9 doi: https://doi.org/10.29375/01240781.4324
}

\section{Introducción}

Colombia es el principal país receptor de la diáspora venezolana. Desde el inicio de este proceso migratorio el departamento de Santander y, en particular, el Área Metropolitana de Bucaramanga (AMB), han sido uno de los territorios con mayor concentración de población migrante proveniente de Venezuela, por esta razón, desde en el año 2017 en el Instituto de Estudios Políticos (IEP), de la Universidad Autónoma de Bucaramanga (UNAB), se creó el Programa de Acción Universitaria para la Migración (PAUM), que busca promover desde los ámbitos de acción universitaria (docencia, investigación y extensión), la reflexión y el compromiso de inclusión frente a la migración transnacional en Colombia y Santander, desde una perspectiva de derechos y en articulación con los actores implicados en el tema a nivel académico, político, jurídico, social y económico.

Una de las apuestas del componente investigativo del PAUM, ha sido desde el 2018 la caracterización de la población migrante proveniente de Venezuela y residente en el AMB, como insumo de línea pase para la toma de decisiones de política pública, así como de orientación en materia de inversión de recursos provenientes de la cooperación internacional, que favorezcan la gestión integral de la migración a nivel local y regional. En este artículo se presentan los resultados de esta apuesta.

\section{Metodologia}

La caracterización se sustenta en el paradigma analítico de la investigación social, con un enfoque cuantitativo, orientado por el análisis multidimensional que contempla un estudio detallado de las variables que caracterizan a las personas que han adelantado un proceso migratorio desde Venezuela hasta el AMB, dentro de las que se encuentran nacionales venezolanos, nacionales colombianos que han retornado y colombo-venezolanos radicados por más de tres meses en alguna de las cuatro ciudades que integran el AMB.

Hasta la fecha se han realizado cuatro caracterizaciones anuales entre 2018 y 2021 , a través de un muestreo no probabilístico. En 2018, 2019 y 2020 se caracterizaron personas radicadas en Bucaramanga, Piedecuesta, Girón y Floridablanca, mientras que, en 2021, por las restricciones de movilidad impuestas por la pandemia de la COVID-19, solo se caracterizaron personas radicadas en Bucaramanga. 
Tabla 1. Distribución de la muestra

\begin{tabular}{ccccccccc}
\multirow{2}{*}{ Ciudad } & \multicolumn{2}{c}{$\mathbf{2 0 1 8}$} & \multicolumn{2}{c}{$\mathbf{2 0 1 9}$} & \multicolumn{2}{c}{$\mathbf{2 0 2 0}$} & \multicolumn{2}{c}{$\mathbf{2 0 2 1}$} \\
\cline { 2 - 9 } & $\mathbf{N}$ & $\mathbf{9}$ & $\mathbf{N}$ & $\mathbf{\%}$ & $\mathbf{N}$ & $\mathbf{\%}$ & $\mathbf{N}$ & $\mathbf{\%}$ \\
\hline Bucaramanga & 958 & $95 \%$ & 460 & $46 \%$ & 204 & $41 \%$ & 504 & $100 \%$ \\
\hline Floridablanca & 40 & $4 \%$ & 240 & $24 \%$ & 136 & $27 \%$ & 0 & $0 \%$ \\
\hline Girón & 10 & $1 \%$ & 160 & $16 \%$ & 101 & $20 \%$ & 0 & $0 \%$ \\
\hline Piedecuesta & 1 & $0 \%$ & 140 & $14 \%$ & 59 & $12 \%$ & 0 & $0 \%$ \\
\hline Total & $\mathbf{1 0 0 9}$ & $\mathbf{1 0 0} \%$ & $\mathbf{1 0 0 0}$ & $\mathbf{1 0 0} \%$ & $\mathbf{5 0 0}$ & $\mathbf{1 0 0 \%}$ & $\mathbf{5 0 4}$ & $\mathbf{1 0 0 \%}$
\end{tabular}

Fuente 1. Elaboración propia.

En cada una de estas caracterizaciones se aplicó una encuesta estructurada en once dimensiones de análisis: (i) aspectos demográficos; (ii) condiciones en el país de origen; (iii) características del proceso migratorio; (iv) condiciones en la ciudad de acogida; (v) perfil educativo; (vi) perfil laboral; (vii) vinculación laboral en Colombia; (viii) generación de ingresos; (ix) condiciones de salud; (x) respuesta pública al fenómeno migratorio e (xi) inclusión social. En el 2021 se adicionaron dos dimensiones, una referente al impacto de la pandemia en las condiciones de vida de los migrantes y otra sobre cultura política. Los datos obtenidos fueron tabulados, ordenados y procesados en una matriz de doble entrada, para luego ser analizados a partir de la estadística descriptiva e inferencial.

\section{Principales resultados}

\section{a) Aspectos demográficos}

En lo referente al género, en 2018 el 50\% de las personas encuestadas pertenecía al género femenino, en 2019 este porcentaje aumentó ligeramente a $52 \%$ y en 2021 a $61 \%$. En cambio, en 2020 se redujo a $46 \%$. Con respecto a la edad, en todos los años se evidenció que la población que migró desde Venezuela hacia el AMB era joven y se encontraba en edad económicamente activa. En el año 2018 el 88\% de los encuestados se ubicaron en el rango de edad de 18 a 35 años, en 2019 el 80\% y en 2020 el $71 \%$.

En cuanto a la nacionalidad, en todos los años la mayoría de los migrantes tenían exclusivamente la nacionalidad venezolana. En 2018, 2019 y 2021 el $89 \%$ de los encuestados tenía esta nacionalidad y en 2020 el $87 \%$. Además, entre el $1 \%$ y $2 \%$ de los encuestados se encontraba adelantando los trámites para obtener la nacionalidad colombiana y, entre el $8 \%$ y el 9\% de ellos poseía tanto la nacionalidad colombiana como la venezolana.

\section{b) Caracteristicas del proceso migratorio}

La mayoría de los encuestados migró a territorio colombiano en los años 2018 y 2019 (79\%) y el 2020 fue el año que registró los más bajos ingresos (7\%). El ingreso al país, en el periodo de observación, se realizó principalmente por tierra por la ciudad de Cúcuta, Norte de Santander (95\% en el 2018, $87 \%$ en el 2019 y $73 \%$ en 2020). Adicionalmente, los migrantes reportaron haber pagado a personas desconocidas como "trocheros" y guardias venezolanos para ingresar a territorio colombiano a través de pasos fronterizos no autorizados $150 \%$ en $2019,45 \%$ en 2020 y $30 \%$ en 2021 ) y, el $14 \%$ de ellos informó haber sido víctimas de hurto durante su proceso migratorio en los años 2020 y 2021).

\section{c) Condiciones en la ciudad de acogida}

La razón por la que los migrantes eligieron destino final el AMB se asocia con dos factores principalmente: la cercanía y la existencia de redes de apoyo (64\% y $50 \%$ en el 2018 , y $57 \%$ y $23 \%$ en el 2019). En los 3 primeros años las expectativas de permanencia de los encuestados en esta región de Colombia no eran muy claras, dado que la mayoría indicó que planeaba permanecer por un tiempo $(49,5 \%$ en $2018,51 \%$ en 2019 y $58 \%$ en 2020). Por el contrario, en 2021 el $71 \%$ afirmó que

REFLEXIÓN POLÍTICAAÑO 23 Nº 48 DICIEMBRE DE 2021 ISSN (en línea) 2590-8669; IEP - UNAB (COLOMBIA) 
proyectaba quedarse indefinidamente, reflejando así su vocación de permanencia en el territorio. En conexidad con lo anterior, el $74 \%$ de los encuestados en 2018 y el $76 \%$ en el 2020 , afirmó que regresaría a Venezuela si la situación política, económica y social mejora. Sin embargo, en 2021 este porcentaje disminuyó considerablemente a $29 \%$.

En relación con el estatus migratorio, se destaca que en el 2018 el $24 \%$ de los encuestados no tenía ningún documento de identificación, porcentaje que aumentó a $51 \%$ en el 2019, y se redujo al $29 \%$ en 2020 y al $8 \%$ en 2021 . Es importante anotar que, entre 2018 y 2021 hubo un aumento considerable en el número de personas portadoras del Permiso Especial de Permanencia (PEP), documento de regularización migratoria, que pasó de poseerlo el 9\% de los encuestados en 2018 al $79 \%$ en 2021.

Con respecto a las condiciones de residencia de los encuestados en 2018 y 2019 el $50 \%$ y $34 \%$, respectivamente, vivían en condición de calle o no tenían un lugar de residencia fija. En cambio, en 2020 este porcentaje fue del 6\% y en 2021 del 1\%. Ante esto, el arrendamiento de casas o apartamentos por parte de los migrantes aumentó, pues pasó del 39\% en 2018 y $32 \%$ en 2019 al 59\% en 2020 y 75\% en 2021. En 2018 el 32\% de los encuestados vivía con más de diez personas y en 2019 el 15\%. En 2020 el 70\% de los migrantes compartía su habitación con entre una y cuatro personas y, en 2021 el 76\% manifestó vivir con entre una y seis personas.

En lo que refiere a la integración con la sociedad de acogida, se identificó que en los encuestados aumentó la percepción de discriminación en su contra. En 2018 sólo el 23\% afirmó haber sido discriminado por nacionales colombianos, en 2019 este porcentaje se incrementó al $45 \%$, en 2020 al $50 \%$ y en 2021 al 57\%.

\section{d) Vinculación laboral}

En los cuatro años la mayoría de los encuestados trabajaba en el sector informal de la economía (55\% en $2018,60 \%$ en $2019,72,6 \%$ en 2020 y $54 \%$ en 2021). Por tanto, un porcentaje reducido contaba con contrato de trabajo ( $3 \%$ en $2018,4 \%$ en 2019 , $5,8 \%$ en 2020 y $10 \%$ en 2021). Cabe anotar que, tanto en el sector formal como informal, la mayoría no trabajaba en el área de formación adquirida en Venezuela $(63 \%$ en $2018,59 \%$ en $2019,70 \%$ en 2020 y $73 \%$ en 2021 ).
Los encuestados también reportaron ser víctimas de explotación laboral en el país (14\% en 2018, $11 \%$ en 2019 y 30\% en 2021). Las principales modalidades de explotación reportadas fueron: a) pago en especie; b) menor pago que a colombianos que realizaban la misma actividad; c) prestación del servicio sin recibir un salario sino amenazas relacionadas con alertar a las autoridades migratorias; d) asignación de jornadas de trabajo superiores a las ocho (8) horas diarias, sin pago de horas extras, dominicales y festivos, así como devengando un salario inferior al mínimo legal vigente.

\section{e) Generación de ingresos}

La mayoría de los migrantes encuestados ganaba menos de un salario mínimo legal mensual vigente (55\% en 2018, 53\% en 2020 y $61 \%$ en 2021 ). Así mismo, enviaban mensualmente remesas a Venezuela (58\% en 2018, 30\% en 2019, $94 \%$ en 2020 y 35\% en 2021). Lo anterior, en razón a que tenían personas dependientes económicamente. En 2018, 2019 y 2020 un porcentaje considerable tenía entre 1 y 3 personas dependientes $(47 \%, 60 \%$ y $47 \%$, respectivamente), mientras que en 2021 la mayoría contaba con entre 1 y 4 personas dependientes (52\%). El monto mensual de estas remesas osciló entre 20.000 y 120.000 pesos colombianos.

\section{f) Aspectos de salud}

En 2018 y 2020 casi la totalidad de los encuestados no se encontraban afiliados al Sistema de Seguridad Social en Salud (95\% y 75\%, respectivamente). En 2021 el panorama cambió en este aspecto, pues el 79\% afirmó estar afiliado, lo cual está íntimamente relacionado con el porcentaje de personas portadoras del PEP. Adicionalmente, menos del 10\% de los encuestados tenía alguna discapacidad ( $1 \%$ en 2018 , $3 \%$ en 2019 , 8,8\% en 2020 y $4 \%$ en 2021 ). La mayoría de encuestados indicó estar en condiciones óptimas de salud ( $80 \%$ en 2018 , $82 \%$ en 2019 y $81,8 \%$ en 2020). En materia de salud sexual y reproductiva, se encontró que la mayoría no utilizaba ningún método de planificación familiar (66\% en 2018, 80\% en 2019 , $69 \%$ en 2020 y $57 \%$ en 2021 ).

\section{g) Respuesta pública al fenómeno migratorio}

La mayoría de los encuestados afirmó que nunca había recibido ayudas gubernamentales durante su residencia en el país ( $89 \%$ en $2018,88,2 \%$ en 
2020 y $54 \%$ en 2021). No obstante, en tiempos de pandemia un porcentaje considerable (46\%), indicó que sí obtuvo asistencia humanitaria por parte de entidades públicas, organismos internacionales y ONGs a través de mercados, bonos económicos y ayudas para el emprendimiento.
En todos los años predominó en la población encuestada el desconocimiento sobre las rutas de atención y las instituciones a las que podían dirigirse para acceder a orientación migratoria, atención médica, asesoría jurídica y ayuda humanitaria (Tabla 2).

Tabla 2. Nivel de desconocimiento sobre asuntos de interés

\begin{tabular}{ccccc} 
Asunto & $\mathbf{2 0 1 8}$ & $\mathbf{2 0 1 8}$ & $\mathbf{2 0 1 8}$ & $\mathbf{2 0 1 8}$ \\
\hline Problemas migratorios & $75 \%$ & $65 \%$ & $73,4 \%$ & $10 \%$ \\
\hline Complicaciones de salud & $66 \%$ & $62 \%$ & $66 \%$ & $24 \%$ \\
\hline Ayuda humanitaria & $89 \%$ & $60 \%$ & $85,2 \%$ & $70 \%$ \\
\hline Asesoría jurídica & $92 \%$ & $74 \%$ & $90,6 \%$ & $80 \%$ \\
\hline Defensa de DDHH & $95 \%$ & $83 \%$ & $93 \%$ & $84 \%$ \\
\hline Problemas domésticos o laborales & $90 \%$ & $83 \%$ & ---- & $85 \%$
\end{tabular}

Fuente 1. Elaboración propia

\section{Conclusiones}

En la población migrante proveniente de Venezuela $\mathrm{y}$ residente en el AMB no existen desigualdades significativas por razón de género. Este es un grupo poblacional en su mayoría joven, con óptimas condiciones de salud y vocación de permanencia en el territorio, por lo que representa para la región un bono demográfico significativo, así como una potencial fuerza laboral y emprendedora que puede contribuir al desarrollo y crecimiento económico local.

Es evidente la evolución favorable que ha presentado la población migrante en materia de regularización de su estatus migratorio, condiciones habitacionales y acceso a salud, asî como a ayudas gubernamentales y beneficios derivados de la cooperación internacional. No obstante, los desafios al respecto son aún bastante amplios, dadas las condiciones de vulnerabilidad económica e irregularidad laboral en la que se encuentra la población. Así mismo, se identificó que los migrantes provenientes de Venezuela son altamente vulnerables a ser víctima del delito y la discriminación antes, durante y después de su proceso migratorio, así como las barreras de acceso a la ruta de atención, dado el desconocimiento que predomina frente a estas y las instituciones, servicios y derechos a los que pueden acceder.

\section{Referencias}

Bonilla, M. E. y Tobón, M. (2019). Migrantes venezolanos en el Área Metropolitana de Bucaramanga. Informe Mayo, 2019. Bucaramanga, Colombia: Universidad Autónoma de Bucaramanga (UNAB). Recuperado de https://repository.unab. edu.co/bitstream/handle/20.500.12749/12353/2019\%20 Migracion\%20Venezolana\%20Presentacion.pdf?sequence $=2$ \&isAllowed $=y$

Bonilla, M. E., Tobón, M., Botero, D., Sandoval, J. y Zambrano, E. (2020). Características de los migrantes provenientes de Venezuela en el AMB. Informe abril 2020. Bucaramanga, Colombia: Universidad Autónoma de Bucaramanga (UNAB). Recuperado de https://repository.unab. edu.co/bitstream/handle/20.500.12749/12354/2020\%20 Migracion\%20Venezolana\%20Presentacion.pdf?sequence $=1$ \&isAllowed $=y$

Bonilla, M. E., Pardo, M., Pérez Guevara, N., Botero, D., Sandoval, J. y Zambrano, E. (2021). Migrantes provenientes de Venezuela en Bucaramanga. Informe Marzo, 2021. Bucaramanga, Colombia: Universidad Autónoma de Bucaramanga (UNAB). Recuperado de https://repository.unab. edu.co/bitstream/handle/20.500.12749/12466/2021_ Informe_investigacion_Caracterizacion.pdf?sequence $=1$ \&isAllowed $=y$ 